\title{
Combined multimodal cerebral monitoring and focused hemodynamic assessment in extremely low birth weight infants - Potential benefits or potential costs?
}

Israel NIDCAP Training Center, Meir Medical Center, Kfar-Saba, Israel, affiliated to Sackler School of Medicine,

Tel-Aviv University, Tel-Aviv, Israel. NIDCAP \& Science Sub-Committee, NFI

Target article: Combined Multimodal Cerebral Monitoring and Focused Hemodynamic Assessment in the First $72 \mathrm{~h}$ in Extremely Low Gestational Age Infants. Deshpande P, Jain Ríos DR, Bhattacharya S, Dirks J, Baczynski M, McNamara KP, Hahn C, McNamara PJ, Shah P, Guerguerianb AM. Neonatology 2020; 117:504-512. DOI: 10.1159/000508961

B rain injury remains one of the major unresolved challenges in neonatal care. With improvements in the overall survival rate of newborn infants, it was natural that attention would shift to intact survival and good neurodevelopmental outcomes. It is widely acknowledged that neonatal brain injury is the result of the complex interaction between pathological processes, the developmental trajectory, genetic susceptibility, and environmental influences. Hence, no single intervention can have a significant effect on outcomes for the infant. Rather, a coordinated, interdisciplinary approach combined with precise clinical care is needed to facilitate new neuroprotective approaches to the phenomenon. This understanding has been translated into several concepts of neonatal care. Two distinct examples are the "Small Baby Unit"1 and the "Neuro-Intensive Care Nursery" ("Neuro NICU"). ${ }^{2}$ The two NICU modalities advocate for the implementation of uniformly standardized care guidelines, adherence to evidencebased practices, and the creation of comprehensive and multidisciplinary teams. However, while the target of the "Small Baby Unit" is the unique population of Extremely Low Gestational Age (ELGA) infants, the target population of the "Neuro NICU" includes all newborns at risk of brain damage, including full-term infants with Hypoxic Ischemic Encephalopathy (HIE) or suffering from strokes or seizures, as well as ELGA infants. Furthermore, the concept of the "Small Baby Unit" focuses on optimization of care for these uniquely vulnerable infants in the context of parent-centered care principles. The "Neuro NICU", for its part, relies on the use of advanced technology such as modern neuromonitoring and neuroimaging, as described in the target article. So, despite the common goal of improving developmental outcomes, the two models for preterm infant care differ greatly.

Severe intraventricular hemorrhage (IVH) and white matter injury are associated with a significant risk of adverse neurodevelopmental outcomes and remain a persistent challenge for preterm infants. The first 72 hours after birth present with the greatest risk of brain injury and is considered "the critical window" for intervention and preventing neurological damage. Many quality-improvement (QI) projects aimed at preventing IVH focus on this critical period. The study by Deshpande et al., "Combined Multimodal Cerebral Monitoring and Focused Hemodynamic Assessment in the First $72 \mathrm{~h}$ in Extremely Low Gestational Age Infants", is a prospective observational cohort study investigating the feasibility and safety of advanced cerebral and hemodynamic monitoring in this population of vulnerable infants during this sensitive period.

\section{Study Details}

Fifty infants born between $23^{+0}$ and $27^{+6}$ weeks gestation were enrolled on the study. The infants had all been managed using an IVH prevention bundle, which included midline head positioning and minimal handling. Cerebral regional oxygen saturation $\left(\mathrm{CrSO}_{2}\right)$ and integrated EEG (aEEG) were measured continuously, and echocardiography (ECHO) and head ultrasounds were performed 2-4 times. The small head size, fragile skin, coexisting headgear with an interface for ventilatory support, and high ambient incubator humidity all made sensor application challenging. However, the study demonstrated that the combined monitoring approach was feasible with 98\% of the infants. Mild erythema without skin breakdown beneath $\mathrm{CrSO}_{2} 2$ sensors was noted in $8 / 50$ subjects (16\%), and desaturations were reported during $17 / 197(8.6 \%)$ of the ultrasound studies. Compared to infants with no IVH, infants with severe IVH (grade III/IV) showed a different pattern of cerebral and systemic hemodynamics. Specifically, changes detected by NearInfrared Spectroscopy (NIRS) showed low $\mathrm{CrSO}_{2}$ and high cerebral fractional tissue extraction, consistent with physiological changes related to hypoxia-reperfusion - known to be one of the risk factors for IVH. ${ }^{3}$ However, since the study was not designed to identify specific variables predicting IVH, along with the low incidence of severe IVH (8\%), the clinical relevance of this observation could not be assessed. The authors concluded that cerebral and cardiovascular multimodal monitoring for ELGA infants is safe and well-tolerated, with a low adverse event rate.

\section{Technological Advances}

The technological development of non-invasive bedside techniques over recent years has allowed the bedside monitoring of heart function and hemodynamics by functional ECHO, 
cerebral oxygenation by NIRS, as well as cerebral electrical activity by aEEG. This type of monitoring can support clinicians in identifying infants at risk of IVH, to allow for early neuroprotective interventions.

Functional echocardiography and NIRS have been used as clinical and research tools to assess changes in systemic and cerebral blood flow and oxygenation during transitional circulation in preterm infants. It has been documented that both cerebral blood flow and cerebral oxygenation are lower for infants who develop IVH3. However, it is yet to be determined whether monitoring cerebral oxygenation, if combined with clinical interventions when cerebral oxygenation levels are outside the desired range, can prevent cerebral injury and improve neurological outcomes. A recent multicenter randomized clinical trial, the $\mathrm{SafeBoosC},{ }^{4}$ demonstrated that it was possible to reduce the burden of cerebral hypoxia during the first 72 hours of life using treatment guidelines for respiratory and hemodynamic support. However, despite reductions in cerebral hypoxia for less than half of the treatment group, there were no differences in brain injury markers between the groups: neither in the aEEG tracing, nor in specific blood biomarkers. ${ }^{5}$ The authors questioned the significance of cerebral hypoxia as an etiology for brain injury, recommending that the guidelines should not be used outside a research setting. ${ }^{5}$

Functional echocardiography can provide direct assessment of hemodynamics at the bedside and can be used as a modern hemodynamic monitoring tool in the neonatal intensive care unit. This is currently regarded by many clinicians as an extension to the infant clinical examination in conditions such as neonatal hypotension and shock, suspected patent ductus arteriosus, and suspected persistent pulmonary hypertension of the newborn. ${ }^{6-10}$ The anatomic, physiological, and hemodynamic information provided by a functional $\mathrm{ECHO}$ can be used in directing specific interventions and evaluating response to treatment. ${ }^{6}$ It has been shown that a functional $\mathrm{ECHO}$, when carefully performed by a trained neonatologist, is well tolerated even by extremely preterm infants. ${ }^{7}$ In combination with blood pressure measurement, it can provide essential information about the hemodynamic status of the newborn and enables a targeted hemodynamic management approach that can account for the underlying pathophysiologic mechanisms of circulatory failure in the individual patient. ${ }^{6-9}$ Toyoshima et al. demonstrated in a prospective cohort study that tailor-made circulatory management significantly reduced both the incidence and the severity of IVH, improved survival rates, and was associated with a trend towards a decrease in mental retardation. ${ }^{9}$ An echocardiographic assessment of the hemodynamic status of the newborn has the potential to improve neonatal intensive care; however, there is still a paucity of prospective studies demonstrating improved outcomes.

The combined monitoring also included recordings of an aEEG and a head ultrasound. Both are used mainly to estimate the timing of the brain injury and its severity, to improve prognosis prediction. ${ }^{10}$ Changes in cerebral oxygenation during head ultrasounds were reported previously for more than half
FIGURE 1. Premie HAL manikin (Gaumard' scientific) with a $\mathrm{CrSO}_{2}$ sensor on the forehead and 3 aEEG sensors on the scalp, as described by Deshpande et al.

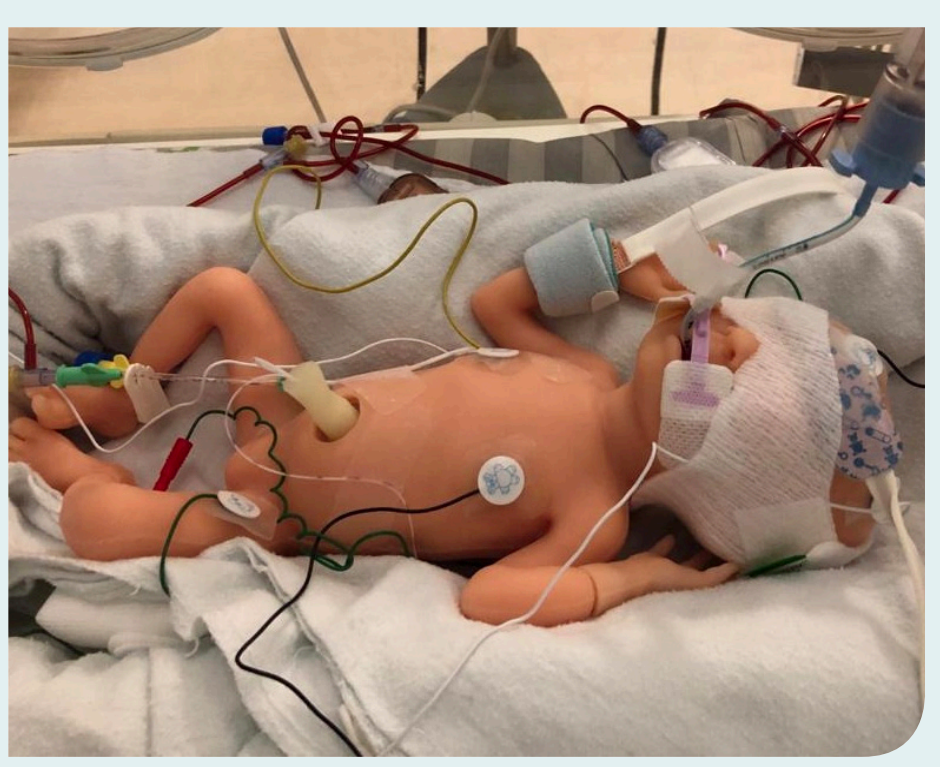

of the infants; ${ }^{11}$ indeed, QI projects aiming for neuroprotection recommend postponing the first ultrasound until 72 hours after birth. ${ }^{12}$

There is more to multimodal cerebral monitoring, as pointed out by Deshpande et al. The illustration in Fig. 1 presents compelling proof of the possible impact of multimodal monitoring on bonding processes and parental stress - both recognized as factors that can affect long-term neurodevelopment.

\section{Implications for NIDCAP}

There is a significant body of evidence showing the positive effects of sensitive and responsive maternal behavior on child development. Higher levels of early maternal responsiveness, sensitivity and positive mind-set have been positively associated with infants' cognitive and social-emotional development, supporting the proposition that healthy early mother-infant interactions can improve not only behavioral but also developmental outcomes ${ }^{13}$.

The first hours after birth represent a crucial period: not only in a physiological sense, due to the hemodynamic instability and transitional processes involved in it, but also because this is a sensitive period for the mother-child interaction, setting the basis for subsequent maternal behavior.

Close contact between mother and child in the first hours after birth is essential, to provide the optimal conditions for the maternal behavior that will facilitate secure attachment in the first year of life. Separation following birth restricts opportunities for the mother-infant dyad to engage in intimate physical contact, potentially altering the unfolding of the affectionate bond between mother and infant. In very low birth weight preterm infants, close contact between mother and child is not routinely possible. The technical environment of the baby and the architecture of NICUs pose additional barriers to physical closeness. This separation hampers normal physical contact and emotional closeness between the parents and their infant, with long-lasting 
consequences for emotional programming, neurodevelopmental outcomes, and parental mental health. ${ }^{13,14}$

Several studies have reported differences in the bonding behavior of the mothers of preterm and full-term infants. Mothers of preterm infants were found to show less secure attachment, as well as less acceptance of the infant and reduced caregiving sensitivity ${ }^{12,13}$ Feldman et al. ${ }^{14}$ showed that the delayed first contact between mother and child led to the decrease of maternal attachment behaviors and representations. Mehler et al. ${ }^{15}$ demonstrated that mothers who were able to see their infant in the first 3 hours after birth developed a more optimal maternal attachment. Thus, strategies to facilitate bonding, despite the obstacles posed by the infant's neurobehavioral immaturity and medical challenges, are an imperative in the NICU setting. Moreover, these strategies need to be anticipated and intentional, designed by and considered from the different perspectives that a multidisciplinary and well-coordinated team can provide.

For parents, the extreme preterm delivery of their infant will be a traumatic experience, one followed by a phase of extreme psychological disturbance. Many parents feel detached and separated from their infants as they cannot or do not feel able to interact as freely with their infant as they would with a full-term infant at home. A recent meta-analysis underlines the fact that parents may be extremely distressed and disturbed by the sight of their baby attached to tubes and equipment, and by the fragile appearance of extreme preterm infants. Indeed, the stress related to the physical appearance of their infant was found to be the second greatest source of burden for parents; ${ }^{16}$ so, it is of concern that the extra tapes and tubing of the multimodal monitoring approach may be an additional source of distress for both mothers and fathers.

Future studies must include an assessment of the impact of multi-modal monitoring on opportunities for early bonding, and on parental stress.

\section{Personal Reflection}

I should disclose that my insights on the article by Deshpande et al. are influenced by my perspective as a Neonatologist and NIDCAP trainer. After a 30-year professional career, and with the equanimity and wisdom that retirement allows, I truly believe that the biggest achievement in my career was being part of the transformative processes that my own NICU went through, to offer individualized and supportive care for infants and families in the framework of the NIDCAP Nursery Program. Therefore, until more evidence is available, my answer to the first question - does combined multimodal cerebral monitoring poses potential benefits or potential cost? - is probably individualization. That is to say, individualized monitoring and individualized care in the context of family-centered care. ${ }^{17}$ Accordingly, I will probably advocate for the development of "small baby units" over "Neuro NICUs", because they allow for the appropriate family-centered developmental care that can address the different needs of extreme preterm infants. There is a growing body of evidence suggesting that family-centered and developmental care strategies have the potential to improve neurodevelopmental outcomes, and that these need to be considered as an essential part of future neuroprotection care bundles. ${ }^{17}$

References

1. Fathi O, Nelin LD, Shepherd EG, Reber KM. Development of a small baby unit to improve outcomes for the extremely premature infant. Journal of Perinatology. 2021, 12:1-8. doi: 10.1038/s41372-021-00984-0.

2. Austin T. The development of neonatal neurointensive care. Pediatric Research. 2019, 12-18. doi: 10.1038/s41390-019-0729-5

3. Lim J, Hagen E. Reducing Germinal Matrix-Intraventricular Hemorrhage: Perinatal and Delivery Room Factors. NeoReviews. 2019; 20(8): e452-e463. doi: 10.1542/neo.20-8-e452

4. Pellicer A, Greisen G, Benders M, et al. The SafeBoosC phase II randomized clinical trial: a treatment guideline for targeted near-infrared-derived cerebral tissue oxygenation versus standard treatment in extremely preterm infants. Neonatology 2013; 104:171-8. doi:10.1159/000351346

5. Plomgaard AM, van Oeveren W, Petersen TH, et al. The SafeBoosC II randomized trial: treatment guided by near-infrared spectroscopy reduces cerebral hypoxia without changing early biomarkers of brain injury. Pediatric Research. 2016; 79(4):528-35. doi: 10.1038/pr.2015.266

6. Tissot C, Singh Y. Neonatal functional echocardiography. Opinion in Pediatrics. 2020; 32(2):235-244. doi: 10.1097/MOP.

7. de Boode WP, van der Lee R, Horsberg Eriksen B et a.l European Special Interest Group 'Neonatologist Performed Echocardiography' (NPE). The role of Neonatologist Performed Echocardiography in the assessment and management of neonatal shock. Pediatric Research. 2018; 84(Suppl 1):57-67. doi: 10.1038/ s41390-018-0081-1.

8. Giesinger RE, McNamara PJ. Hemodynamic instability in the critically ill neonate: an approach to cardiovascular support based on disease pathophysiology. Seminars in Perinatology. 2016; 40(3):174-88. doi: 10.1053/j.semperi.2015.12.005

9. Toyoshima K, Kawataki M, Ohyama M et al. Tailor-made circulatory management based on the stress-velocity relationship in preterm infants. Journal of For mos Medical Association. 2013; 112(9):510-7. doi: 10.1016/j.jfma.2013.02.011.

10. Pisani F, Spagnoli C. Monitoring of newborns at high risk for brain injury. Italian Journal of Pediatrics. 2016; 14;42(1):48. doi: 10.1186/s13052-016-0261

11. Murthy P, Zein H, Thomas S. Neuroprotection Care Bundle Implementation to Decrease Acute Brain Injury in Preterm Infants. Pediatric Neurology, 2020; 110:42-48. doi: 10.1016/j.pediatrneurol.2020.04.016

12. Forcada-Guex M, Pierrehumbert B, Borghini A et al. Early dyadic patterns of mother-infant interactions and outcomes of prematurity at 18 months. Pediatrics. 2006,118(1): e107-14. doi: 10.1542/peds.2005-1145.

13. Korja R, Latva R, Lehtonen L. The effects of preterm birth on mother-infant interaction and attachment during the infant's first two years. Acta Obstetrics Gynecology Scandinavia. 2012; 91(2):164- 73. doi: 10.1111/j.16000412.2011.01304.x.

14. Feldman R, Weller A, Leckman JF, Kuint J, Eidelman AI. The nature of the mother's tie to her infant: maternal bonding under conditions of proximity, separation and potential loss. Journal of Child Psychology Psychiatry. 1999; 40: 929-939. PMID: 10509887.

15. Mehler K, Wendrich D, Kissgen R, et al. Mothers seeing their VLBW infants within $3 \mathrm{~h}$ after birth are more likely to establish a secure attachment behavior: evidence of a sensitive period with preterm infants? Perinatology. 2011, 31(6):404-10. doi: 10.1038/jp.2010.139.

16. Caporali C, Pisoni C, Gasparini L, Ballante E, Zecca M, Orcesi S, A global perspective on parental stress in the neonatal intensive care unit: a meta-analytic study. Provenzi L. Journal of Perinatology. 2020, 40(12):1739-1752. doi: 10.1038/s41372-020-00798-6.

17. Soni R, Tscherning Wel-Wel C, Robertson NJ. Neuroscience meets nurture: challenges of prematurity and the critical role of family-centred and developmental care as a key part of the neuroprotection care bundle. Archives of Disease in Childhood, Fetal Neonatal Edition. 2021 fetalneonatal-2020-319450. doi: 10.1136/archdischild 